\title{
Norsolorinic Acid Mutant of Aspergillus flavus
}

\author{
By K. E. PAPA \\ Department of Plant Pathology, University of Georgia, Athens, Georgia 30602, U.S.A.
}

(Received 2 September 1981; revised 17 November 1981)

A mutant of Aspergillus flavus accumulating norsolorinic acid and approximately $50 \%$ less aflatoxin than the parent strain was recovered after treatment of conidia with $\mathrm{N}$ methyl- $N^{\prime}$-nitro- $N$-nitrosoguanidine. The gene nor controlling this mutant phenotype was recessive and linked to afl-1 and leu on linkage group VII. Diploids homozygous for nor were similar to haploids in norsolorinic acid accumulation. The analysis of recombinant diploids and haploids showed that $a f-1$ and nor were both distal to leu on the same chromosome arm.

\section{INTRODUCTION}

Aflatoxins, secondary metabolites produced by Aspergillus flavus and Aspergillus parasiticus, have attracted considerable attention, and much has been reported about their biosynthesis and biological effects. Several intermediates in the aflatoxin biosynthetic pathway have been established through studies of induced mutants (Hsieh et al., 1976). Lee et al. (1971) recovered a mutant of $A$. parasiticus which accumulated norsolorinic acid and less aflatoxin than the wild-type. This mutant was recessive in diploids (Bennett, 1979). Several aflatoxin mutants having no detectable pigmented intermediates have been recovered in A. flavus (Papa, 1979).

This paper describes the genetic analysis of a norsolorinic acid mutant of A. flavus, including linkage detection and mapping.

\section{METHODS}

Strains. Five auxotrophic strains of $A$. flavus having either white $(w)$ or $\tan (t)$ spore colour were used in this study. Their individual genotypes were as follows: 1. $w$ lys (requiring lysine); 2. $t$ pdx leu (pyridoxine, leucine); 3. $t$ leu; 4. afl-1 $t$ leu; and 5. $t$ arg (arginine). Strain 4 was also impaired in aflatoxin production (afl-1). The induction of auxotrophy, spore colour variation, and aflatoxin mutants has been described previously (Leaich \& Papa, 1974; Papa, 1976, 1979).

Following the exposure of conidia from strain 1 to $N$-methyl- $N^{\prime}$-nitro- $N$-nitrosoguanidine (NG), a mutant, designated 1-N and having an orange-red mycelium, was recovered. The gene controlling the production of this orange-red pigment (norsolorinic acid) has been designated nor (Bennett, 1979).

Media. The complete medium (CM) consisted of Czapek-Dox broth, 0.75\% (w/v) malt extract, $0.25 \%(\mathrm{w} / \mathrm{v})$ yeast extract and $1.5 \%(\mathrm{w} / \mathrm{v})$ agar. The minimal medium (MM) was $\mathrm{CM}$ without the malt and yeast extracts. Diploids were plated on $\mathrm{CM}$ containing $0.007 \%(\mathrm{w} / \mathrm{v})$-fluorophenylalanine to induce haploidization. Appropriately supplemented $\mathrm{MM}$ was used for the identification of nutritional requirements of haploid segregants. The medium for production of aflatoxin consisted of $2 \%(w / v)$ yeast extract and $20 \%(w / v)$ sucrose.

Aflatoxin and norsolorinic acid determinations. Aflatoxin and norsolorinic acid were extracted according to procedures described by Lillard et al. (1970) and Papa (1976). Extracts were suspended in chloroform, appropriately diluted, and spotted on thin-layer chromatographic plates (pre-coated silica gel) from EM Laboratories, Elmsford, N.Y., U.S.A. Chromatograms for aflatoxin and norsolorinic acid separations were developed in chloroform/acetone $(88: 12, \mathrm{v} / \mathrm{v})$ and benzene/acetic acid $(95: 5 \mathrm{v} / \mathrm{v})$, respectively. Initially, amounts of aflatoxin were quantified using a Turner model 111 fluorometer. Later, only visual estimates were made by observing the intensity of fluorescence of extracts on thin-layer plates. In some cases extracts were merely scored 
as aflatoxin positive or negative. The aflatoxin standard was obtained from Aldrich Chemical Co., Milwaukee, Wis., U.S.A.

Norsolorinic acid could usually be detected in chloroform extracts; however, when total extraction was desired, acetone extraction procedures described by Bennett (1979) were followed. Extracts were usually scored as norsolorinic acid positive or negative. A standard was prepared from a norsolorinic acid-producing mutant of A. parasiticus (ATCC 24690) isolated by Bennett \& Goldblatt (1973). The authenticity of norsolorinic acid from our A. flavus mutant was checked by comparing extracts with the standard on a DuPont 860 high-pressure liquid chromatograph. Several mutants impaired in aflatoxin production and not accumulating norsolorinic acid served as controls.

Genetic analysis. Techniques involved in forcing heterokaryons, recovering diploids, and subsequently haploidizing diploids were the same as those described by Papa (1976). For linkage detection, parasexual crosses were made between mutant $1-\mathrm{N}$ and haploid strains 2, 3, 4, and 5. A total of 418 haploid segregants (117, 103, 115 , and 83 from diploids involving strains $2,3,4$, and 5, respectively) were recovered and the segregation of nor and other gene markers was determined.

Diploids homozygous for nor, resulting from mitotic recombination, were identified from the appearance of orange-red sectors on plates of heterozygous diploids. Sectors were selected and subcultured on CM until they were uniformly pigmented. Twenty diploids and 10-15 haploid segregants from each of 17 of the diploids were tested for nutritional requirements. Each diploid and all haploids auxotrophic for leucine were also tested for aflatoxin production. Based on the distribution of genes within the same linkage group, the sequence of the genes in relation to the centromere was determined.

\section{RESULTS AND DISCUSSION}

A mutant accumulating an orange-red pigment was recovered following NG treatment of conidia from a white-spored lysine auxotroph of $A$. flavus. By comparing extracts from this mutant to a standard, it was concluded that the accumulating pigment was norsolorinic acid. Identical $R_{F}$ values were obtained for the orange-red pigments of this nor mutant and the standard in both chromatography systems (thin-layer and high-pressure liquid). The nor mutant accumulated approximately $50 \%$ less aflatoxin than the parental strain. A significant reduction in aflatoxin accumulation was also noted in a nor mutant of $A$. parasiticus (Lee $e t$ al., 1971).

Diploids (1-N)/2, (1-N)/3, and (1-N)/5 formed between $1-\mathrm{N}$ (nor $w$ lys) and haploid strains 2,3 , and 5 were aflatoxin positive. The (1-N)/4 diploid, heterozygous for the dominant aflatoxin mutant afl-1 (Papa, 1980), however, did not accumulate aflatoxin. None of the diploids accumulated norsolorinic acid, thus indicating that nor was recessive. Similarly, nor-1 in A. parasiticus was recessive in diploids (Bennett, 1979).

Haploidization of diploids provided evidence for linkage of nor to leu and afl-1 (Table 1). Linkage between leu and afl-1 on linkage group VII was previously reported (Papa, 1979). No recombinants were obtained between nor and leu among 335 haploid segregants or between nor and afl-1 among 115 segregants. The other gene markers ( $w, \arg , l y s$, and pdx) segregated independently of nor. Mutant nor and its wild-type allele did not necessarily segregate in 1:1 ratio among the haploids recovered from each diploid; however, overall, the ratio $(191: 227)$ was not significantly different from $1: 1$ at the 0.05 level of probability. Leucine prototrophs apparently had a selective advantage among segregants from diploid $(1-\mathrm{N}) / 4$. Consequently, only $10 / 115$ segregants were nor.

Twenty diploids homozygous for nor were recovered following mitotic recombination in diploid (1-N)/4. Each diploid was aflatoxin positive and prototrophic for leucine. Since afl-1 is dominant over its wild-type allele, each nor/nor diploid was obviously also homozygous for the wild-type allele of afl-1 (Table 2). Of 17 diploids haploidized, eight (Type A) yielded 118 prototrophs and nine (Type B) yielded 50 prototrophs and 47 auxotrophs. Type A diploids were apparently homozygous and type B diploids heterozygous for the leucine locus. All haploid segregants were nor and those auxotrophic for leucine were all aflatoxin positive. Leucine prototrophs were not tested for aflatoxin. 
Table 1. Linkage detection of norsolorinic acid mutant nor among haploid segregants from four diploids

\begin{tabular}{lccr} 
& \multicolumn{3}{c}{ No. of haploid segregants } \\
\cline { 2 - 4 } Diploid & Total & nor & nor $^{+}$ \\
$(1-\mathrm{N}) / 2$ & 117 & 81 & 36 \\
$(1-\mathrm{N}) / 3$ & 103 & 42 & 61 \\
$(1-\mathrm{N}) / 4$ & 115 & 10 & 105 \\
$(1-\mathrm{N}) / 5$ & 83 & 58 & 25 \\
Total & 418 & 191 & 227
\end{tabular}

\begin{tabular}{cccccc}
\multicolumn{5}{c}{ Frequency of recombination with nor (\%) } \\
\hline leu & lys & $w$ & $p d x$ & afl-1 & arg \\
0 & 64 & 50 & 54 & - & - \\
0 & 22 & 31 & - & - & - \\
0 & 12 & 70 & - & 0 & - \\
- & 40 & 31 & - & - & 31
\end{tabular}

Table 2. Determination of gene order for nor, afl-1, and leu on linkage group VII

\begin{tabular}{lrl}
\multicolumn{1}{c}{ Culture } & No. & \multicolumn{1}{c}{ Genotype } \\
Heterozygous diploid $(1-\mathrm{N}) / 4$ & 1 & + nor $+/$ afl $-1+$ leu \\
Recombinant diploids: & & \\
$\quad$ Type A & 8 & + nor $+/+$ nor + \\
Type B & 9 & + nor $+/+$ nor leu \\
Haploid segregants from: & & \\
Type A & 118 & + nor + \\
Type B & 50 & + nor + \\
& 47 & + nor leu
\end{tabular}

Mitotic recombination provides a means for determining the sequence of genes on a chromosome (Pontecorvo \& Kafer, 1958). Since heterozygous gene loci distal to both the centromere and the point of crossing-over can become homozygous, it was concluded that afl-1 and nor were distal to leu on the same chromosome arm. Recombination occurred between leu and the centromere in eight diploids and distal to leu in nine diploids. Since crossing-over was not detected between afl-1 and nor it was not certain which one was closest to leu. However, unless afl-1 and nor are closely linked, the results of this study, based on the selection of nor/nor homozygotes, support the sequence afl-1 nor leu centromere.

Nevertheless, selection for nor/nor diploids may be misleading because of the relationship of afl-1 and nor in the same biosynthetic pathway. Bennett et al. (1971) \& Bennett (1981) demonstrated the concomitant production of aflatoxin and norsolorinic acid. If afl-1 is epistatic to nor, the only detectable diploids homozygous for nor would be those not carrying the dominant afl-1 allele. This could also account for the apparent lack of afl-1 nor haploid recombinants; nor could not be expressed in the presence of afl-1.

These results indicate that the gene controlling norsolorinic acid accumulation can be useful in mapping studies because the orange-red pigment is easily identified. Mapping aflatoxin genes, however, may present special difficulties.

Appreciation is expressed to Louise Miller for her capable technical assistance.

\section{REFERENCES}

BenNetT, J. W. (1979). Aflatoxins and anthraquinones from diploids of Aspergillus parasiticus. Journal of General Microbiology 113, 127-136.

BENNETT, J. W. (1981). Loss of norsolorinic acid and aflatoxin production by a mutant of Aspergillus parasiticus. Journal of General Microbiology 124, $429-432$.

Bennett, J. W. \& Goldblatt, L. A. (1973). The isolation of mutants of Aspergillus flavus and $A$. parasiticus with altered aflatoxin producing ability. Sabouraudia 11, 235-241.

Bennett, J. W., Lee, L. S. \& Vinnett, C. (1971). The correlation of aflatoxin and norsolorinic acid production. Journal of the American Oil Chemists Society 48, 368-370.

Hsieh, D. P. H., Lin, M. T., YAo, R. C. \& Singh, R. (1976). Biosynthesis of aflatoxin: conversion of norsolorinic acid and other hypothetical inter- 
mediates into aflatoxin $\mathrm{B}_{1}$. Journal of Agricultural and Food Chemistry 24, 1170-1174.

LEAICH, L. L. \& PAPA, K. E. (1974). Aflatoxins in mutants of Aspergillus flavus. Mycopathologia et mycologia applicata 52, 223-229.

Lee, L. S., Bennett, J. W., Goldblatt, L. A. \& Lundin, R. E. (1971). Norsolorinic acid from a mutant strain of Aspergillus parasiticus. Journal of the American Oil Chemists Society 48, 93-94.

LillaRd, H. S., Hanlin, R. T. \& Lillard, D. A. (1970). Aflatoxigenic isolates of Aspergillus flavus from pecans. Applied Microbiology 19, 128-130.
PapA, K. E. (1976). Linkage groups in Aspergillus flavus. Mycologia 65, 1201-1205.

PAPA, K. E. (1979). Genetics of Aspergillus flavus: complementation and mapping of aflatoxin mutants. Genetical Research 34, 1-9.

PAPA, K. E. (1980). Dominant aflatoxin mutant of Aspergillus flavus. Journal of General Microbiology 118, 279-282.

Pontecorvo, G. \& KAFER, E. (1958). Genetic analysis by means of mitotic recombination. $A d$ vances in Genetics 9, 71-104. 